\title{
Enhancement of CdSSe QD exciton luminescence efficiency by hydrogen RF plasma treatment
}

\author{
V.P. Kunets, N.R. Kulish, V.V. Strelchuk, A.N. Nazarov, A.S. Tkachenko, V.S. Lysenko, \\ M.P. Lisitsa \\ Institute of Semiconductor Physics, NAS of Ukraine, 45 prospect Nauky, 03028 Kyiv, Ukraine \\ Phone: +38 (044) 2656282; Fax: +38 (044) 2658342; E-mail:vl_kunets@yahoo.com
}

\begin{abstract}
We report an enhancement of exciton luminescence in $\mathrm{CdS}_{\mathrm{X}} \mathrm{Se}_{1-\mathrm{x}} \mathrm{QD}$ embedded into borosilicate glass matrix and then treated by the low-temperature hydrogen RF plasma. Results clearly confirm the essential crushing of the surface levels that have a high nonradiative recombination efficiency.
\end{abstract}

Keywords: quantum dots, photoluminescence, surface passivation, RF plasma treatment.

Paper received 25.02.03; accepted for publication 16.06.03.

\section{Introduction}

Since the pioneering works by G. Bret [1], V. Borodulin [2] and M. Lisitsa [3], CdSSe-doped glasses have been extensively studied in two directions. Besides investigations of the quantum confinement effects in CdSSe QDs, they were widely tested as the nonlinear-optical materials that can be employed in optical devices. That is why during the early years much interest had been focused on measurements of the absorption saturation threshold $\left(\mathrm{I}_{l}\right)$ [4], the Kerr susceptibility $\chi^{(3)}[5]$ and the carrier lifetime $\tau[6]$ that are the basic parameters in the field. Meanwhile, the experimental data on the lifetime seem to be inconsistent and sometimes contradictory varying from $10 \mathrm{ps}$ to $10 \mathrm{~ns}$ and even more. The following investigations were then directed on the lifetime size dependence [7], and the reasonable explanation based on the role of the surface traps was proposed [8]. On the other hand, the exciton luminescence efficiency does not exceed several percents in these glasses, which is obviously caused by a high nonradiative recombination efficiency of the surface levels. The thermal annealing at high temperatures $\left(\sim 700^{\circ} \mathrm{C}\right)$, which is usually used to anneal the deep levels in composite structures can not be used to modify QD surface, because it stimulates the growth of QDs producing the new dangling bonds and the surface traps for the carriers. Therefore, to use the CdSSe-doped glasses in the nonlinear optical devices [9], one should have the low temperature method to modify the surface states con- trolling the lifetime and the exciton luminescence. In this paper, we report the results about the low temperature hydrogen RF plasma treatment of CdSSe-doped glasses that clearly evidence the crushing of the surface levels rising the exciton lifetime and luminescence efficiency.

\section{Experiment}

CdSSe-doped glasses with different QD's composition were studied at room temperature. The samples were excited with a CW Ar ${ }^{+}$laser $(\lambda=488.0 \mathrm{~nm}, P<100 \mathrm{~mW})$. The PL light was monitored with DFS-24 spectrometer equipped with a photomultiplier and a photon counting system. First order Raman LO spectra were used to control QD composition. The luminescence spectra were measured using the samples prepared by the typical heattreatment procedure [10] and the same samples but after their RF plasma treatment. The reactor of the diode type (the pressure in the chamber was $5 \times 10^{-2}$ Torr, the frequency was $13.6 \mathrm{MHz}$ ) was used. The RF treatment with the power at $1.5 \mathrm{~W} / \mathrm{cm}^{2}$ and additional heating of the sample up to $200^{\circ} \mathrm{C}$ for $30 \mathrm{~min}$ has been performed. These power and additional heating correspond to the sample temperature of about $300^{\circ} \mathrm{C}[11]$. This temperature was chosen to avoid the QDs growth. At these regimes the hydrogen atoms diffuse into the dioxide layer down to $400 \mathrm{~nm}$ [12]. Thus, we can suggest that in the CdSSe-doped glasses with QD concentration up to $10^{14}-10^{15} \mathrm{~cm}^{-3}$ the distance of hydrogen penetration will have a similar order, which 
is sufficient for PL measurements. In fact, the borosilicate glass matrix has the so-called "free" or "vacant" volume, which is associated with its porous structure and can consist of $0.01 \div 0.10$ for various glasses. Thus, we think the real hydrogen penetration depth is greater (about several microns), because the hydrogen ions can diffuse along the porous surfaces in this case.

\section{Results and discussion}

Fig. 1 shows the PL spectra of $\mathrm{CdS}_{\mathrm{x}} \mathrm{Se}_{1-\mathrm{x}}$ QDs before (curve 1) and after (curve 2) RF plasma treatment. All spectra were recorded at the same experimental conditions. The rise of the signal is clearly observed all over the investigated spectral region. Any valuable shift of the maximum was no observed. The halfwidth of the luminescence band was also remained practically invariable in the range of the measurement accuracy. The plasma stimulated enhancement of the PL signal was determined to be about several dozens of percents and could rise in some cases up to $70 \%$. All the experimentally observed data are summarized in Table 1.

The relatively wide PL peaks in Fig. 1 are attributed to the direct electron-hole recombination in $\mathrm{CdS}_{\mathrm{X}} \mathrm{Se}_{1-\mathrm{X}}$ QDs, which is also confirmed by the superlinear PL signal dependence on the excitation intensity. The short wavelength shift of the peaks is attributed to both the composition and the confinement of the electrons and holes in QDs. In Fig.1 the exciton peaks are only presented. The large halfwidth of the peak is particularly explained by the size dispersion of QDs. The average radius $\bar{r}$ of QD's in our samples were less than the Bohr exciton radius in the bulk $\left(a_{B} \sim 3 \div 5 \mathrm{~nm}\right)$. In this strong confinement regime (the confinement energy was 150 to $200 \mathrm{meV}$ ), the electron-hole pair in QD ("squeezed pair" or "exciton") is considered as the elementary electron QD excitation. The short wavelength laser excitation (488.0 $\mathrm{nm}$ ) was used to excite the low-energy confined states in QDs and to avoid the photoionization effects [13]. In general, PL intensity is described by the formula:

$I_{P L}=I_{0}\left\lfloor 1-(1-R)^{2} \exp (-K d)\right\rfloor \eta^{*} \eta_{i n}$,

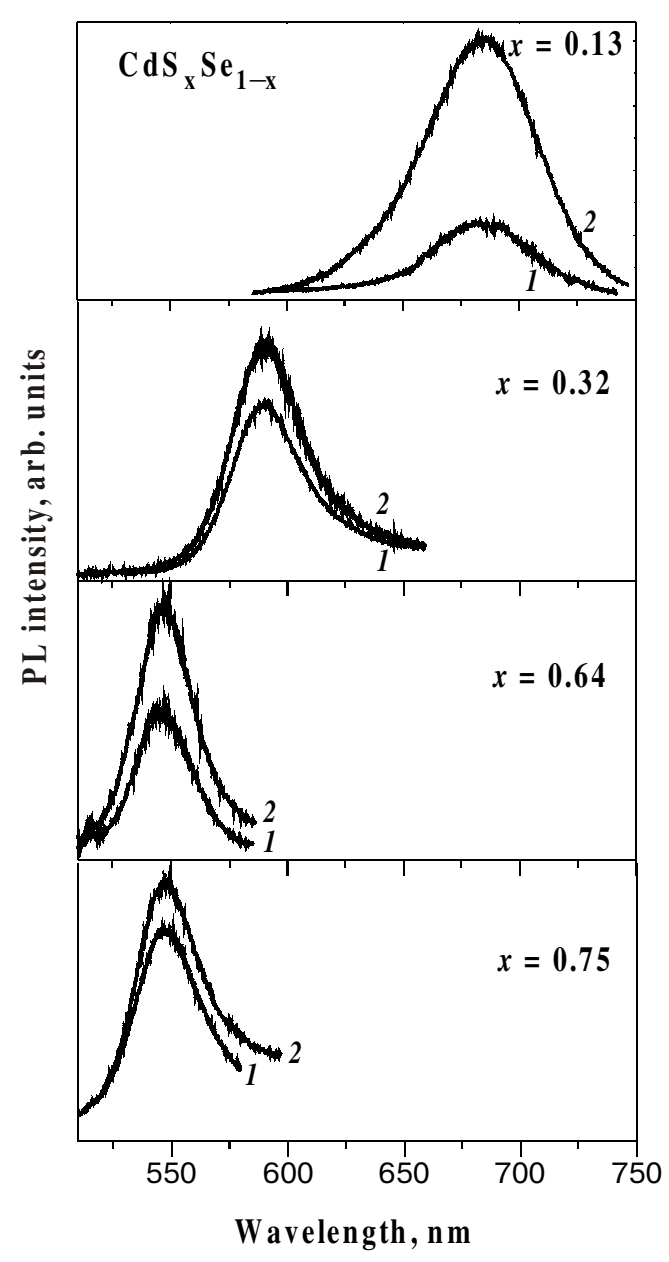

Fig. 1. Exciton PL spectra of $\mathrm{CdS}_{\mathrm{X}} \mathrm{Se}_{1-\mathrm{x}}$ QDs at room temperature before (1) and after (2) RF plasma treatment.

where $R$ is the reflection coefficient, $K$ is the absorption coefficient, $d$ is the sample thickness, $\eta_{i n}$ is the internal luminescence quantum yield, $\eta^{*}$ describes the luminescence losses in the structure due to the light scattering, reabsorption, etc. The value $\eta_{i n}$ is related to the carrier lifetime or radiative recombination time $\tau_{R}$ by the following formula:

Table 1. Sample and PL spectra parameters for four compositions.

\begin{tabular}{|c|c|c|c|c|c|c|c|c|}
\hline Parameter $\quad$ Composition & \multicolumn{2}{|c|}{$\mathrm{CdS}_{0.13} \mathrm{Se}_{0.87}$} & \multicolumn{2}{|c|}{$\mathrm{CdS}_{0.32} \mathrm{Se}_{0.68}$} & \multicolumn{2}{|c|}{$\mathrm{CdS}_{0.64} \mathrm{Se}_{0.36}$} & \multicolumn{2}{|c|}{$\mathrm{CdS}_{0.75} \mathrm{Se}_{0.25}$} \\
\hline Average radius, $\bar{r}, \mathrm{~nm}$ & \multicolumn{2}{|c|}{$7.80[10]$} & \multicolumn{2}{|c|}{$2.50[10]$} & \multicolumn{2}{|c|}{$2.35[10]$} & \multicolumn{2}{|c|}{$2.65[10]$} \\
\hline QD concentration, $N, \mathrm{~cm}^{-3}$ & \multicolumn{2}{|c|}{$\sim 10^{15}[10]$} & \multicolumn{2}{|c|}{$5.3 \cdot 10^{13}[10]$} & \multicolumn{2}{|c|}{$2.8 \cdot 10^{14}[10]$} & \multicolumn{2}{|l|}{$\sim 10^{13}[10]$} \\
\hline PL spectra parameters & $\begin{array}{l}\text { before } \\
\text { RF-treat. }\end{array}$ & $\begin{array}{l}\text { after } \\
\text { RF-treat. }\end{array}$ & $\begin{array}{l}\text { before } \\
\text { RF-treat. }\end{array}$ & \begin{tabular}{|l|} 
after \\
RF-treat.
\end{tabular} & $\begin{array}{l}\text { before } \\
\text { RF-treat. }\end{array}$ & $\begin{array}{l}\text { after } \\
\text { RF-treat. }\end{array}$ & $\begin{array}{l}\text { before } \\
\text { RF-treat. }\end{array}$ & $\begin{array}{l}\text { after } \\
\text { RF-treat. }\end{array}$ \\
\hline PL max., nm & $685.0 \pm 0.5$ & $684.5 \pm 0.5$ & $589.4 \pm 0.5$ & $589.9 \pm 0.5$ & $546.5 \pm 0.5$ & $546.8 \pm 0.5$ & $546.1 \pm 0.5$ & $547.1 \pm 0.5$ \\
\hline PL halfwidth, eV & 0.192 & 0.190 & 0.131 & 0.131 & 0.147 & 0.148 & 0.121 & 0.123 \\
\hline PL intensity, arb.un. & 1.00 & 1.70 & 1.00 & 1.24 & 1.00 & 1.18 & 1.00 & 1.39 \\
\hline
\end{tabular}




\section{V.P. Kunets et al.: Enhancement of CdSSe QD exciton luminescence ...}

$\eta_{\text {in }}=\tau_{S} /\left(\tau_{R}+\tau_{S}\right) \approx \tau_{S} / \tau_{R}$

where $\tau_{S}$ - is the carrier lifetime determined by the nonradiative recombination processes. It is taken into account in (2) that usually in QDs $\tau_{S}<<\tau_{R}$. This fact is clearly confirmed by both the data on the absorption saturation [9] and the low external PL quantum yield $\left(\eta_{\text {in }} \eta^{*} \sim 1 \%\right)$. For the "exciton" luminescence

$\eta_{\text {in }} \approx \tau_{S} / \tau_{E X}=B n / \sigma_{S} v N_{S}$

where $\tau_{E X}=1 / B n$ is the exciton lifetime or radiative exciton recombination time, $B$ is the coefficient of the bimolecular recombination, $n$ is the exciton concentration, $\tau_{S}=1 / \sigma_{S} v N_{S}, \sigma_{S}$ is the cross section for the carriers captured by the surface traps, $v$ is the thermal velocity of the carriers, and, finally, $N_{S}$ is the concentration of the surface traps that are the effective non-radiative recombination levels for the electron or holes in these small QDs [9].

The $B$ coefficient does not depend on the excitation intensity and describes the probability of the bimolecular recombination. As a rule, $B, \sigma_{S}$ and $v$ do not also depend on $N_{\mathrm{S}}$. On the other hand, only two possible ways can be considered to increase of $\eta_{\text {in }}$ (see, formula (3)): the increasing of the exciton concentration $(n)$ which recombine radiativelly, while fixing $N_{\mathrm{S}}$ value, or the decreasing of $N_{\mathrm{S}}$ value at the same excitation conditions. In our case, we intentionally tried to change the value of $N_{\mathrm{S}}$ using the RF plasma treatment of the samples and measuring the PL spectra under the same conditions. This allowed us to rise drastically the $\eta_{\text {in }}$ (see, formula (3)) and, finally, the external luminescence yield $\eta_{\text {in }} \eta^{*}$ at the constant losses $\eta^{*}$ that is experimentally observed as the enhancement of the exciton PL intensity. In general, we realize that the non-radiative recombination efficiencies for electrons and holes are quite different, and that the surface modification drastically changes $\tau_{S}$ value probably for one type of the carrier only. Meanwhile, the photoionization of QD which modifies the charge of the $\mathrm{E}_{1}{ }^{-}$-centers in the glass matrix near the QD surface [13] can also effect on $\tau_{S}$. To develop the model of the $\mathrm{CdS}_{\mathrm{x}} \mathrm{Se}_{1-\mathrm{x}}$ QD surface modification by the RF plasma treatment one should perform additional investigations. At the same time, we also realize that the effect is, one as the matter of fact, more strong than we've visually observed. Indeed, the glass thickness modified by the hydrogen atoms is smaller than the absorption light depth. In this case, the PL signal is formed by the whole excited region of the glass, while the thin hydrogen riched layer of the glass gives only to the effect.

\section{Conclusions}

We prepared CdSSe QD with the modified surface using the low temperature RF hydrogen plasma treatment. In these samples, we have observed the enhancement of the exciton luminescence in comparison with the non-treated samples. Using the simple idea about the mechanism of the exciton luminescence in QDs, we have concluded that such treatment decreases the quantity of the nonradiative recombination centers on QD surface. This result clearly demonstrates the possibility of the effective QD surface modification in these composite materials.

\section{Acknowledgments}

This work has been partially supported by the joint project "Physics of Solid State Nanostructures" between the Ukrainian State Foundation of Fundamental Researches and the Russian Foundation for the Basic Researches.

\section{References}

1. G. Bret, F. Gires. Giant-pulse laser and light amplifier using variable transmission coefficient glasses as light switches // Appl. Phys. Let. 4 (10), pp.175-176 (1964).

2. V.I. Borodulin, N.A. Yermakova, L.A. Rivlin and V.S. Shildyaev. Emission of mono-pulses of coherent light by a two-component medium with negative absorption // Zhurnal Eksperimentalnoj i Teoreticheskoj Fiziki 48 (3), pp.845-849 (1965) (in Russian).

3. M.P. Lisitsa, N.R. Kulish, V.I. Geets and P.N. Koval. Modulation of laser quality by means of KS-19 filters // Optika $i$ Spektroskopiya 20 (3), pp.508-510 (1966) (in Russian).

4. M.P. Lisitsa, N.R. Kulish, P.N. Koval and V.I. Geets. An influence of laser radiation on the transparency of KS-19 glass // Optika i Spektroskopiya 21 (6), pp.1721-1729 (1966).

5. L.H.Acioli, A.S.L.Gomes, J.R.Rios Leite. Measurement of high-order optical nonlinear susceptibilities in semiconductor-doped glasses // Appl. Phys. Lett. 53 (19), N11, pp.17881790 (1988)

6. V.S.Williams, G.R.Olbright, B.D.Fluegel, S.W.Koch, N.Peygnambarian. Optical nonlinearities and ultrafast carrier dynamics in semiconductor doped glasses // Journal of Modern Optics 35 (12), pp. 1979-1993 (1988).

7. K.Shum, G.C.Tang, M.R.Junnarkar, R.R.Alfano. Electronhole recombination lifetimes in a quasi-zero-dimensional electron system in CdSSe // Appl. Phys. Lett. 51 (22), N11, pp.18391841 (1987)

8. S.V. Kletskij, N.R. Kulish, V.P. Kunets, M.P. Lisitsa, V.N Sokolov. Nonlinear absorption of light by semiconductor microcrystals in glass matrix // Ukrainskij Fizicheskij Zhurnal. 36 (1), pp.18-28 (1991) (in Ukraine)

9. N.R. Kulish, V.P. Kunets, M.P. Lisitsa. Linear and nonlinear optical properties of CdSSe-doped glasses used for optical bistable devices // International Journal of Electronics. $\mathbf{7 8}$ (3), pp.1-6. (1995).

10. N.R.Kulish, V.P.Kunets, M.P.Lisitsa. Determination of semiconductor quantum dots parameters by optical methods // Superlattices and Microstructures. 22 (3), pp.341-351 (1997).

11. A.N. Nazarov, V.I. Kilchitska, I.P. Barchuk, A.S. Tkachenko, S. Ashok. Radio frequency plasma unnealing of positive charge generated by Fowler-Nordheim electron injection in buried oxides in silicon // J. Vac. Sci. Technol.(B) 18 (3), pp.1254-1261 (2000)

12. D. Ballutaud, A. Boutry-Forveille, A. Nazarov. Hydrogen thermal stability in buried oxides of SOI structures // Microelectronic Engineering. 48, pp.359-362 (1999).

13. V. Ya. Grabovskis, Ya.Ya. Dzenis, A.I. Ekimov, I.A. Kudryavtsev, M.N. Tolstoj, U.T. Rogulis. Photoionization of semiconductor nanocrystals in a glass // Fizika Tverdogo Tela. 31 (1), pp. 272-275 (1989) (in Russian). 